\title{
A FULLY-INTEGRATED WEARABLE MICROFLUIDIC ACTUATION AND SENSING PLATFORM FOR BIOMARKER ANALYSIS
}

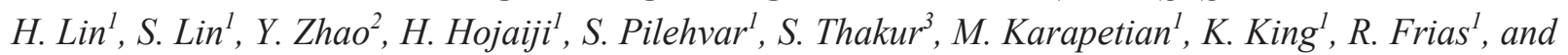 \\ S. Emaminejad \\ ${ }^{1}$ Department of Electrical Engineering and Computer Sciences, University of California, Los Angeles, CA, USA \\ ${ }^{2}$ Department of Materials Science and Engineering, University of California, Los Angeles, CA, USA \\ ${ }^{3}$ Department of Bioengineering, University of California, Berkeley, CA, USA
}

\begin{abstract}
Here, we devise a suite of electro-fluidic and electrothermal interfaces, integrated within a wearable microfluidic platform, to implement an illustrative set of critical biofluid actuation (including pumping and valving) and sensing operations, thus enabling autonomous wearable sample processing and analysis.
\end{abstract}

\section{INTRODUCTION}

Recent advances in electrochemical sensor development, flexible device fabrication, and low power electronics have created an unprecedented opportunity to realize wearable biomarker monitoring platforms that provide frequent and objective measurements of the health condition of individuals. Previously reported wearable biomarker monitoring platforms demonstrated suitable sensing schemes for the detection of target analytes in biofluid samples (e.g. sweat and interstitial fluid) that are passively collected in absorbent pads or microfluidic channels. However, due to the lack of controlled biofluidic handling strategies, their operations cannot be scaled to perform autonomous sample processing and analysis.

\section{RESULTS AND DISCUSSION}

Figure 1 illustrates the system level diagram of our platform's operation. The wearable microfluidic module is integrated into a miniaturized and custom-developed wireless printed circuit board to realize seamless biofluid control and analysis.

To implement pumping, we exploit AC electrothermal flow (ACEF) which is amenable for high conductivity fluid handling (e.g., biofluids). ACEF arises in the presence of a non-uniform electric field which results in temperature gradient and fluid motion. Here, we used a pair of asymmetric ACEF electrodes, which creates imbalanced temperature and electric field profiles to break the symmetric competitive vortices, resulting in directionally-induced fluid motion (i.e. pumping) [1], as verified by our COMSOL simulation (Fig. 2a). Our design consists of a narrow electrode (width: $40 \mu \mathrm{m}$ ) and a wide electrode (width: $90 \mu \mathrm{m}$ ), which are separated by $30 \mu \mathrm{m}$ and patterned onto the bottom of the channel to establish the intended non-uniform electric field profile. To validate the pumping functionality, we applied $1.4 \mathrm{~V}_{\mathrm{RMS}}(>1 \mathrm{MHz})$ across the asymmetric electrode pair, and tracked the motion of the fluid, through time-sequential imaging of a group of microbeads suspended in artificial sweat (Fig. 2b). By increasing the applied voltage levels across the pumping electrode pair, we validated that the induced velocity profile is correlated with the fourth power of the applied voltage $\left(R^{2}=0.97\right.$, Fig. $\left.2 \mathrm{c}\right)$, which is in agreement with previous studies [1].

To realize on-chip valving, we exploit a stimuli-responsive hydrogel, specifically, poly(N-isopropylacrylamide) (PNIPAM), which significantly shrinks in response to local temperature increments. The PNIPAM-valve can be opened when desired by heating the hydrogel above its transition temperature. Accordingly, in our approach, we embedded the hydrogel structure into the microfluidic channel, with a programmable microheater, patterned at its bottom. As shown in Fig. 3a, by applying voltage ( 3 V) across the terminals of the heater, we maintained the local temperature above $48{ }^{\circ} \mathrm{C}$, to subsequently shrink the hydrogel and open the microfluidic channel. We validated the functionality of the controlled valving methodology on body by performing human subject testing (Fig. 3b).

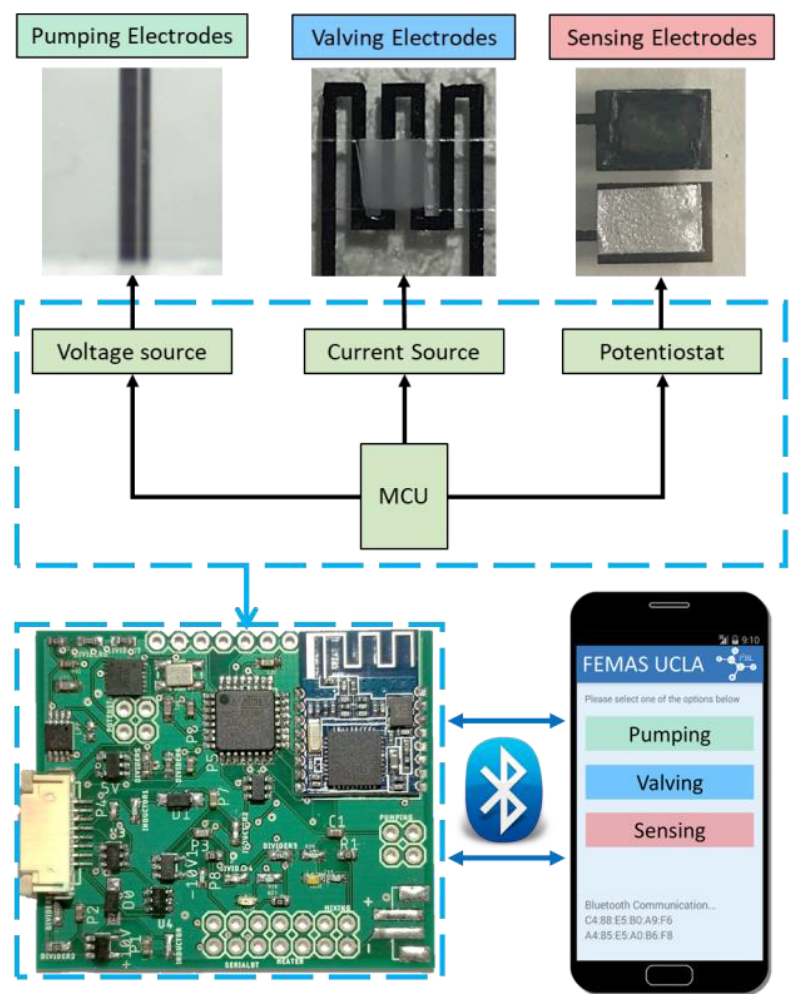

Figure 1: The schematic diagram of our fully-integrated wearable microfluidic platform. The microfluidic module contains: i) an asymmetric electrode pair to induce pumping, ii) an embedded hydrogel/heater configuration to implement valving, and iii) an enzymatic sensing interface for biomarker quantification (e.g. glucose). The system-level operation is achieved through integration of the microfluidic module with a wireless and miniaturized circuit board. The data communication/control commands are communicated via Bluetooth to/from a custom-developed mobile application.

To demonstrate our platform's biomarker sensing capability, we developed (following our previous protocol [2]) and embedded enzymatic sensing electrodes in our microfluidic module to measure the glucose content of sweat samples. As shown in Fig. 4a, the corresponding glucose sensor calibration curve demonstrates high degree of linearity within physiologically relevant range of glucose concentration. 


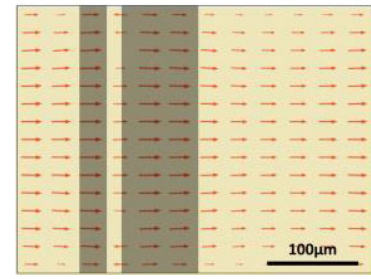

(a)

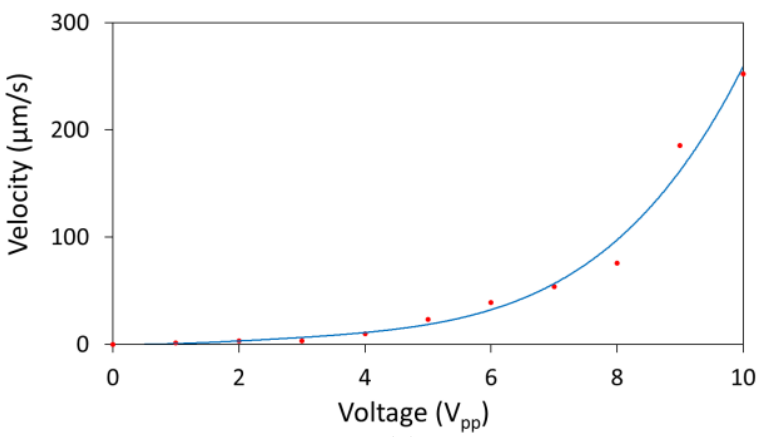

(c)

Figure 2: (a) COMSOL-simulated flow velocity profile induced by a pair of asymmetric electrodes. (b) Time-sequential imaging of artificial sweat pumping. A representative group of beads are tracked as they move unidirectionally from left to right in the time span of $6 \mathrm{~s}$. (c) Measured pumping velocity profile with respect to applied voltage levels.

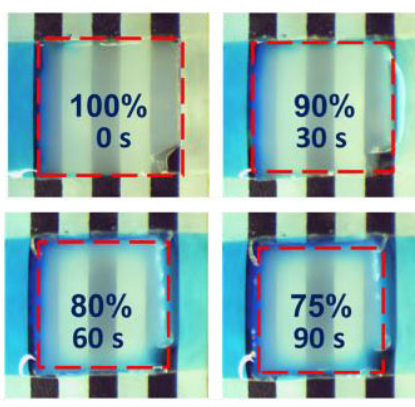

(a)

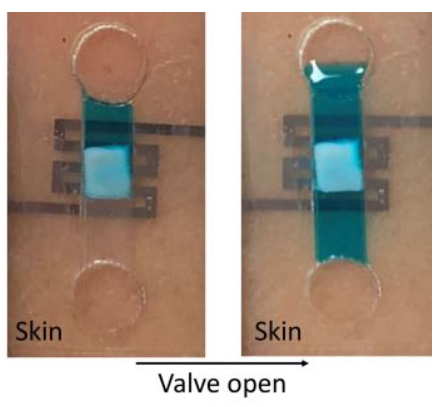

(b)
Figure 3: (a) Timeline of Hydrogel shrinkage upon activating the heater (temperature maintained above $48{ }^{\circ} \mathrm{C}$ ). Blue-dyed artificial sweat passes through the valve from left to right as valve shrinks. (b) Demonstration of on-body valving as controlled by our circuit board.

We validated our glucose sensor functionality by analyzing the iontophoretically-stimulated sweat samples, collected from a subject during 12-h fasting and $0.5 \mathrm{~h}$ after $30 \mathrm{~g}$ glucose intake (Fig.4b). Our results indicate that oral glucose consumption in the fasting state is followed by increased sweat glucose levels (here, from $254 \mu \mathrm{M}$ to $345 \mu \mathrm{M}$ ).

In conclusion, we developed and validated the functionalities of a fully-integrated wearable actuation and sensing microfluidic platform for autonomous biofluid processing and analysis.
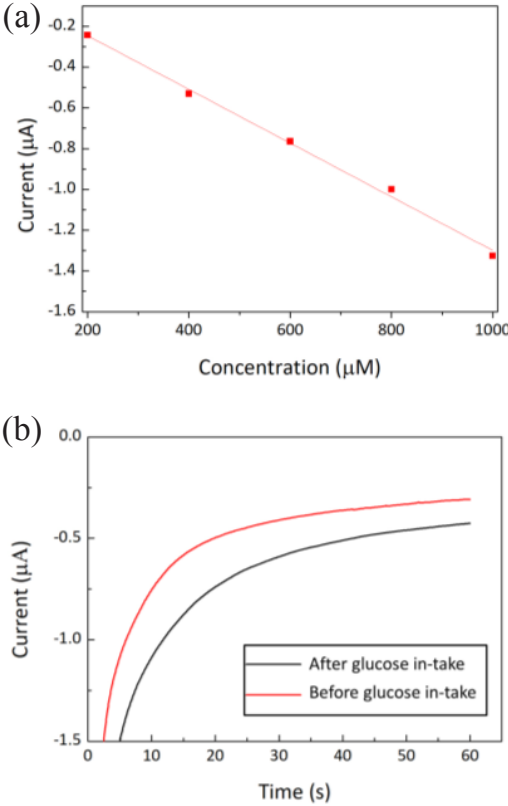

Figure 4: (a) Calibration curve of our developed glucose sensor response (performed in spiked artificial sweat samples). (b) Our sensor response to the sweat samples of a subject during 12-h fasting state and 30 minutes after $30 \mathrm{~g}$ of glucose intake, demonstrating the elevation in sweat glucose level after glucose intake.

\section{REFERENCES}

[1] J. Wu, M. Lian, and K. Yang, "Micropumping of biofluids by alternating current electrothermal effects", Applied Physics Letters 90, 234103 (2007).

[2] S. Emaminejad, W. Gao, H. Y. Y. Nyein, S. Challa, R. W. Davis, A. Javey, "Flexible Systems for Wearable Physiological Monitoring Applications", Solid State Sensors, Actuators, and Microsystems Conference, (Hilton Head), pp. 108-109, June 2016.

\section{ACKNOWLEDGEMENTS}

The authors appreciate the members of UCLA nanoelectronics research facility and Lux Lab from UCLA Library for their help in device fabrication. 